\title{
Review of: "Choroid plexus volume predicts expansion of chronic lesions and brain atrophy"
}

\author{
Christel Weiss ${ }^{1}$ \\ 1 Ruprecht-Karls-Universität Heidelberg
}

Potential competing interests: The author(s) declared that no potential competing interests exist.

Statistics: Please indicate in which cases correlation coefficient according to Pearson or according to Speram has been calculed.

Table 1: For EDSS at baseline and EDSS at follow up median ist presented. What'S the meaning of "1.0 + 0.97 " or "1.0 + 1.3"? Are 0.97 and 1.3 standard deviations? However, it is not senseful to present a median value together with a standard deviation. Interquartile ranges should be given instead.

Lesion volumes: I suppose mean values (or medians?) together with standard deviations are presented. However, these standard deviations are rther high compared to the mean values. This suggests that the distributions are skewd. Again, median values togteher with interquartile ranges should be preferred.

ROC analysis: To which volume do the sensitivity of $85 \%$ and the specificity of $76 \%$ refer?

A forward stepwise linear regression analysis has been performed. Please indicate which parameters have been considered for the basic model? Waht doe it mean "F change $<0.0001$ "? Is this a $p$ value? 